\title{
Short communication: Multidrug-resistant Acinetobacter baumannii-calcoaceticus complex isolated from infant milk formula and utensils in a nursery in Rio de Janeiro, Brazil
}

\author{
B. C. Araújo, M. S. Moraes, L. E. O. Costa, and J. S. Nascimento ${ }^{1}$ \\ Laboratory of Microbiology, Instituto Federal de Educação, Ciência e Tecnologia do Rio de Janeiro, Rua Senador Furtado 121, \\ Maracanã, Rio de Janeiro, CEP 20270-021, Brazil
}

\begin{abstract}
Infant milk formulas are not sterile products, and pathogenic bacteria can survive and multiply in these products. This study was performed, initially, to detect the presence of Salmonella spp. in reconstituted infant milk formula and on utensils previously sanitized used in their preparation or distribution in a nursery of a public hospital in Rio de Janeiro. None of the samples tested carried Salmonella spp. However, further identification of colonies growing on the selective media revealed the presence of several other gram-negative bacteria. Seventeen isolates were identified as belonging to Acinetobacter baumannii-calcoaceticus complex. Fourteen isolates presented a multidrug-resistance profile, by disc diffusion assays, and one of them-JE4 - was also resistant to imipenem. The detection of Acinetobacter isolates in this work demonstrates inadequate hygiene practices in the preparation or distribution of infant milk formula.
\end{abstract}

Key words: Acinetobacter spp., multidrug resistance, infant milk formula, utensil

\section{Short Communication}

Concerns have been raised about the consumption of infant milk formulas (IMF) because the ingestion of contaminated formula or the use of improperly sanitized utensils in their preparation can be mechanisms of acquiring infections (Mammina et al., 2007). Multidrug-resistant gram-negative bacteria represent a major cause of infections in neonatal intensive-care units, and the feeding with infant formula is significantly associated with cross-transmission of resistant strains (Mammina et al., 2007; Mardaneh and Dallal, 2013). Many studies report the presence of different gram-negative bacteria in IMF, including Acinetobacter

Received September 5, 2014.

Accepted January 2, 2015.

${ }^{1}$ Corresponding author: janaina.nascimento@ifrj.edu.br spp., but the attention given to this type of food was, in the most cases, focused only on Cronobacter spp. and Salmonella spp. (Wang et al., 2009; Miled et al., 2010; Abdullah Sani et al., 2013). Salmonella enterica is one of the most worrisome pathogens associated with IMF, because clear evidence exists that their presence can result in severe disease in at-risk population that consumes that food (Arsalan et al., 2013). The initial objective of this study was to detect the presence and the antibiotic resistance profile of Salmonella spp. and other gram-negative bacteria, if any, in reconstituted IMF samples and utensils previously sanitized used in their preparation or distribution from the nursery room of a public hospital in Rio de Janeiro, Brazil.

All the utensils - jars, spoons, baby bottles, trays, and rubber nipples - were sanitized by the nursery staff by washing with soap and water (with the aid of a brush, in the case of bottles) and subsequent immersion in boiling water for $10 \mathrm{~min}$. No other sanitizing agent, such chlorine solution, was used. Reconstituted IMF from 3 different lots made for infants 0 to 6 mo of age were prepared in sterile heat-resistant containers with large volumes and subjected to heating in a conventional microwave oven for $45 \mathrm{~s}$ before distribution. Samples from utensils were obtained by swabs, and samples from IMF were collected in the original small containers. All of them were kept under refrigeration immediately conducted at the laboratory.

For samples of reconstituted IMF, preenrichment, selective enrichment, and plating were performed as described by Normative Instruction 62 (Brazil, 2003). Twenty-five milliliters of the IMF was homogenized with $225 \mathrm{~mL}$ of buffered peptone water $(1 \%$, wt/vol) and incubated for $24 \mathrm{~h}$ at $35^{\circ} \mathrm{C}$. Selective enrichment was done by transferring $1 \mathrm{~mL}$ of this culture to Rappaport-Vassiliadis, tetrathionate, and selenite cystine broths (Himedia, São Paulo, Brazil) and incubating for $24 \mathrm{~h}$ at $41^{\circ} \mathrm{C}$. Broths were then streaked on bismuth sulfite (Himedia), XLD (Himedia), and Rambach (Merck, Kenilworth, NJ) agar plates and incubated for $24 \mathrm{~h}$ at $35^{\circ} \mathrm{C}$. Agar EMB (Himedia) was also included, aiming 
for the isolation of other gram-negative bacteria. Swabs collected were incubated in $9 \mathrm{~mL}$ of $1 \%$ (wt/vol) buffered peptone water at $37^{\circ} \mathrm{C}$ for $18 \mathrm{~h}$ before the selective enrichment and plating procedures at the same agar media described.

Atypical and typical colonies suggestive of Salmonella sp. were selected and submitted to conventional biochemical tests and slide agglutination with somatic polyvalent anti-Salmonella sera (Probac do Brasil, São Paulo, Brazil). The identification was confirmed by using the commercial panel Bactray (Laborclin, São Paulo, Brazil). Salmonella enterica ATCC 19214 was used as control.

Samples from spoons did not present colonies on the media used in this study. A total of 44 isolates were obtained from the other utensils and from the IMF analyzed. Salmonella sp. was not detected; however, different gram-negative species were identified. The presence of microorganisms was expected, because the cleaning of vessels was performed only by washing with soap and hot water immersion. Commercial sodium hypochlorite solution could also have been used. Reconstituted IMF provides an ideal environment for the growth of microorganisms. According to the FAO/ WHO risk assessment (FAO/WHO, 2006), the number of microorganisms is dramatically reduced when IMF is reconstituted with water heated to at least $70^{\circ} \mathrm{C}$. The evidence of contamination presented in this study suggests that, because large volumes are prepared and heated in microwave ovens, the internal temperature does not reach this minimum.

Acinetobacter baumannii-calcoaceticus (ABC) complex was the most isolated bacteria (17 isolates, $37.8 \%$ ), followed by Enterobacter cloacae (12 isolates, 26.7\%). Other species included Hafnia alvei, Escherichia vulneris, Enterobacter aerogenes, and Proteus mirabilis. The ABC complex isolates were found on the jars and also in 2 reconstituted IMF.

In this work, the differentiation of $A$. baumannii from the ABC complex was not performed. Even automated systems such as Phoenix (Becton Dickinson, Franklin Lakes, NJ) and Vitek2 and API 20NE (BioMérieux, Durham, NC) are not able to differentiate the species from the ABC complex (Abbott and Peleg, 2014). Useful tools involving the amplification by PCR of genes from carbapenem-hydrolyzing class $\mathrm{D} \beta$-lactamases and the subunit B from DNA gyrase (bla $a_{\text {OXA-51-like }}$ and $g y r B$ ) can help to identify members of this complex (Higgins et al., 2007; Hamouda, 2010; Gurung et al., 2013) and will be used in a future work.

The results were consistent with a study involving data from 7 countries about microorganisms isolated from IMF and utensils used in its preparation, in which
A. baumannii-calcoaceticus and E. cloacae were the most found microorganisms (Chap et al., 2009).

The ABC complex organisms are found usually in water and soil. However, these microorganisms can cause infections such as pneumonia, urinary tract, surgical wound, and bloodstream infections (Blossom and Srinivasan, 2008; Barsoumian et al., 2013).

These pathogens are gaining importance because of the outbreaks reported and the infections caused in neonates. These patients are, in general, vulnerable, and the drug resistance presented by clinical isolates of this organism constitutes a real threat (Zarrilli et al., 2012).

Because of this fact, the 17 Acinetobacter isolates were submitted to antibiotic susceptibility testing by disc diffusion, performed according to Clinical and Laboratory Standards Institute guidelines (CLSI, 2013). The following antibiotics (Sensifar, São Paulo, Brazil) were employed: amikacin (30 mg), ampicillinsulbactam (10/10 mg), ceftazidime (30 mg), cefotaxime (30 mg), ciprofloxacin $(5 \mathrm{mg})$, gentamicin (10 mg), imipenem $(10 \mathrm{mg})$, tetracycline $(30 \mathrm{mg})$, tobramycin (10 $\mathrm{mg})$, and trimethoprim-sulfamethoxazole (1.25/23.75 mg). Escherichia coli ATCC 29522 was used as control. Results are presented in Table 1.

Resistance to ampicillin-sulbactam was observed in $15(88.2 \%)$ isolates, whereas $14(82.3 \%)$ isolates were resistant to cefotaxime and $12(70.6 \%)$ to trimethoprimsulfamethoxazole. The other tested antibiotics showed lower rates of resistance. Fourteen isolates were resistant to antibiotics belonging to at least 3 different classes, which confer to these bacteria a multidrug-resistant (MDR) profile (Magiorakos et al., 2012; Heizmann et al., 2013).

One of the MDR isolates - JE4 - was also resistant to imipenem, an antibiotic from the carbapenems class. Imipenem and meropenem are considered the most effective antibiotics for the treatment of infections caused by this pathogen, and when the resistance to this class of antibiotics occurs, the treatment options become limited (Karageorgopoulos and Falagas, 2008; Joshi and Litake, 2013). Carbapenem-resistant Acinetobacter baumannii has emerged as a serious threat among ill neonates, because the bacteremia in these cases has a very high mortality rate (Thatrimontrichai et al., 2013).

The increasing of the antimicrobial resistance is providing therapeutic challenges, especially considering that most $A$. baumannii strains that are resistant to the carbapenems are also resistant to the many other antibiotics (Fishbain and Peleg, 2010). Carbapenem resistance may be conferred through various mechanisms, including the expression of lactamase carbapenemases, especially the group of the metallo-lactamases, able 
Table 1. Source and antimicrobial-resistance profile of the isolates of Acinetobacter baumannii-calcoaceticus complex studied in this work

\begin{tabular}{|c|c|c|c|}
\hline Source & Isolate & Resistance profile $^{1}$ & $\begin{array}{l}\text { Growth on MDR } \\
\text { Acinetobacter } \text { agar }^{2}\end{array}$ \\
\hline \multirow[t]{12}{*}{ Jars } & JE2 & SAM, CTX, SXT & + \\
\hline & JE3 & AMI, SAM, CTX & + \\
\hline & JE4 & SAM, CTX, IMP, SXT & + \\
\hline & JE5 & AMI, SAM, CTX, GEN, SXT & + \\
\hline & JE6 & AMI, SAM, CIP, CTX, GEN, TET, SXT & + \\
\hline & JE8 & CIP, CTX, GEN, TOB, SXT & + \\
\hline & JR1 & SAM, CTX, GEN, SXT & + \\
\hline & JR2 & SAM, CIP, CTX, GEN, TOB & + \\
\hline & JR3 & SAM, CTX, TOB, SXT & + \\
\hline & JR4 & AMI, SAM, CTX, GEN, SXT & + \\
\hline & JR5 & SAM, CTX, SXT & + \\
\hline & JR6 & SAM, CTX, SXT & + \\
\hline \multirow[t]{2}{*}{ Baby bottles } & MR1 & AMI, SAM, CTX, SXT & + \\
\hline & ME2 & SAM, CTX, SXT & + \\
\hline $\mathrm{IMF}^{3} 1$ & $\mathrm{AE} 1^{4}$ & SAM & + \\
\hline \multirow[t]{2}{*}{ IMF 2} & $\mathrm{PR} 1^{4}$ & - & - \\
\hline & PR2 & SAM, TET & - \\
\hline
\end{tabular}

${ }^{1} \mathrm{AMI}=$ amikacin; $\mathrm{CTX}=$ cefotaxime; $\mathrm{CIP}=$ ciprofloxacin; $\mathrm{GEN}=$ gentamicin IPM $=$ imipenem; $\mathrm{SAM}=$ ampicillin-sulbactam; SXT = trimethoprim-sulfamethoxazole; TET = tetracycline; TOB = tobramycin.

${ }^{2}+$, growth on selective agar; -, absence of growth or resistance to the antibiotics tested. All the experiments were repeated at least twice. MDR = multidrug resistant. The MDR Acinetobacter agar was from CHROMagar (Paris, France).

${ }^{3} \mathrm{IMF}=$ infant milk formula.

${ }^{4}$ Non-MDR isolate.

to hydrolyze most of the lactams. The production of metallo-lactamases has been described in many Enterobacteriaceae strains and also in Acinetobacter spp. isolates (Maltezou, 2009; Lee et al., 2012). Metallo-lactamases producing $\mathrm{ABC}$ complex strains have become a global concern because these enzymes presented a high hydrolytic activity for the carbapenems, the most important group of antibiotics for the treatment of infections caused by MDR Acinetobacter (Bush and Jacoby, 2010; Roca et al., 2012).

For an additional confirmation, and to seek a practical and rapid method for the detection of MDR Acinetobacter strains in the nursery, a selective chromogenic medium (MDR Acinetobacter, CHROMagar, Paris, France) was used to identify multidrug-resistant isolates by the growth in prominently red colonies, after overnight incubation. All of the 14 multidrug-resistant isolates detected in the disc diffusion assay were also identified as MDR by growth in this medium (Table 1). However, one isolate, AE1, classified as non-MDR, was also able to grow, suggesting that this isolate is probably resistant to other antibiotics not tested in this work. Barsoumian and coworkers (2013) also related that this chromogenic agar permits the rapid detection of MDR Acinetobacter spp., although is unable to distinguish carbapenem-resistant from carbapenemsusceptible strains.

The detection of MDR Acinetobacter isolates in this work demonstrates that inadequate hygiene of the uten- sils could represent a significant risk of the transmission of this multidrug-resistant pathogen to neonates related to the consumption of IMF, reflecting poor infectioncontrol procedures. To solve this problem, educational training emphasizing good manufacturing practices and food safety should be introduced to the staff of the nurseries, aimed at the identification and correction of the critical control points.

\section{ACKNOWLEDGMENTS}

This research was supported by grants from Instituto Federal de Educação, Ciência e Tecnologia do Rio de Janeiro (IFRJ), Brazil, and Fundação de Amparo à Pesquisa do Estado do Rio de Janeiro (FAPERJ), Brazil.

\section{REFERENCES}

Abbott, I. J., and A. Y. Peleg. 2014. Acinetobacter baumannii and hospital infections. Microbiol. Aust. 1:54-56.

Arsalan, A., Z. Anwar, I. Ahmad, A. Saba, S. Baqar, and S. Naqvi. 2013. Microbes in pediatric infant formula. Sci. Nat. 2:116-122.

Barsoumian, A., T. Calvano, A. E. Markelz, R. Cassidy, C. K. Murray, M. L. Beckius, K. Mende, and K. S. Akers. 2013. Variations of CHROMagar Acinetobacter to detect imipenem-resistant Acinetobacter baumannii-calcoaceticus complex. Scand. J. Infect. Dis. 45:446-452. http://dx.doi.org/10.3109/00365548.2013.759270.

Blossom, D. B., and A. Srinivasan. 2008. Drug-resistant Acinetobacter baumannii-calcoaceticus complex: An emerging nosocomial pathogen with few treatment options. Infect. Dis. Clin. Pract. 16:1-13. http://dx.doi.org/10.1097/ipc.0b013e3181635def. 
Brazil. 2003. Ministério da Agricultura, Pecuária e Abastecimento. Resolução da Diretoria Colegiada, n. 62, de 18 de setembro de 2003. Diário Oficial. Federative Republic of Brazil.

Bush, K., and G. A. Jacoby. 2010. Updated functional classification of ß-lactamases. Antimicrob. Agents Chemother. 54:969-976.

Chap, J., P. Jackson, R. Siqueira, N. Gaspar, C. Quintas, J. Park, T. Osaili, R. Shaker, Z. Jaradat, S. H. Hartantyo, N. Abdullah Sani, S. Estuningsih, and S. J. Forsythe. 2009. International survey of Cronobacter sakazakii and other Cronobacter spp. in follow up formulas and infant foods. Int. J. Food Microbiol. 136:185-188.

Clinical and Laboratory Standards Institute (CLSI). 2013. Performance Standards for Antimicrobial Susceptibility Testing: Twenty-Third Informational Supplement, M100-S23. CLSI, Wayne, PA.

FAO/WHO (Food and Agriculture Organization of the United Nations/World Health Organization). 2006. Enterobacter sakazakii and Salmonella in powdered infant formula: Meeting report. FAO/ WHO Microbiological Risk Assessment Series, no. 10. FAO, Rome, Italy.

Fishbain, J., and A. Y. Peleg. 2010. Treatment of Acinetobacter infections. Clin. Infect. Dis. 51:79-84.

Gurung, M., H. M. Nam, M. D. Tamang, M. H. Chae, G. C. Jang, S. C. Jung, and S. K. Lim. 2013. Prevalence and antimicrobial susceptibility of Acinetobacter from raw bulk tank milk in Korea. J. Dairy Sci. 96:1997-2002.

Hamouda, A. 2010. Characterization of epidemiologically unrelated Acinetobacter baumannii isolates from four continents by use of multilocus sequence typing, pulsed-field gel electrophoresis, and sequence-based typing of bla $(O X A$-51-like) genes. J. Clin. Microbiol. 48:2476-2483.

Heizmann, W. R., H. Dupont, P. Montravers, X. Guirao, C. Eckmann, M. Bassetti, M. S. Garcia, M. R. Capparella, D. Simoneau, and K. F. Bodmann. 2013. Resistance mechanisms and epidemiology of multiresistant pathogens in Europe and efficacy of tigecycline in observational studies. J. Antimicrob. Chemother. 68(Suppl. $2): 45-55$.

Higgins, P. G., H. Wisplinghoff, O. Krut, and H. A. Seifert. 2007. PCR-based method to differentiate between Acinetobacter baumannii and Acinetobacter genomic species 13TU. Clin. Microbiol. Infect. 13:1199-1201.

Joshi, S. G., and G. M. Litake., 2013. Acinetobacter baumannii: An emerging pathogenic threat to public health. World J. Clin. Infect. Dis. $3325-36$.

Karageorgopoulos, D. E., and M. E. Falagas. 2008. Current control and treatment of multidrug-resistant Acinetobacter baumannii infections. Lancet Infect. Dis. 8:751-762. http://dx.doi.org/10.1016/ S1473-3099(08)70279-2.

Lee, Y. T., S. C. Kuo, M. C. Chiang, S. P. Yang, C. P. Chen, T. L. Chen, and C. P. Fung. 2012. Emergence of carbapenem-resistant non-baumannii species of Acinetobacter harboring a blaOXA51-like gene that is intrinsic to A. baumannii. Antimicrob. Agents Chemother. 56:1124-1127.
Magiorakos, A. P., A. Srinivasan, R. B. Carey, Y. Carmeli, M. E. Falagas, C. G. Giske, S. Harbarth, J. F. Hindler, G. Kahlmeter, B. Olsson-Liljequist, D. L. Paterson, L. B. Rice, J. Stelling, M. J. Struelens, A. Vatopoulos, J. T. Weber, and D. L. Monnet. 2012. Multidrug-resistant, extensively drug-resistant and pandrug-resistant bacteria: An international expert proposal for interim standard definitions for acquired resistance. Clin. Microbiol. Infect. 18:268-281.

Maltezou, H. C. 2009. Metallo-beta-lactamases in Gram-negative bacteria: Introducing the era of pan-resistance? Int. J. Antimicrob. Agents 33:405.e1-405.e7.

Mammina, C., P. Di Carlo, D. Cipolla, M. Giuffrè, A. Casuccio, V. Di Gaetano, M. R. Plano, E. D'Angelo, L. Titone, and G. Corsello. 2007. Surveillance of multidrug-resistant gram-negative bacilli in a neonatal intensive care unit: Prominent role of cross transmission. Am. J. Infect. Control 35:222-230. http://dx.doi.org/10.1016/j. ajic.2006.04.210.

Mardaneh, J., and M. M. S. Dallal. 2013. Isolation, identification and antimicrobial susceptibility of Pantoea (Enterobacter) agglomerans isolated from consumed powdered infant formula milk (PIF) in NICU ward: First report from Iran. Iran J. Microbiol. 5:263-267.

Miled, R. B., S. Neves, N. Baudouin, B. Lombard, V. Deperrois, P. Colin, and N. G. Besse. 2010. Impact of pooling powdered infant formula samples on bacterial evolution and Cronobacter detection. Int. J. Food Microbiol. 138:250-259. http://dx.doi.org/10.1016/j. ijfoodmicro.2010.01.014

Roca, I., P. Espinal, X. Vila-Farrés, and J. Vila. 2012. The Acinetobacter baumannii oxymoron: Commensal hospital dweller turned pan-drug-resistant menace. Front Microbiol. 3:148 http://dx.doi. org/10.3389/fmicb.2012.00148.

Abdullah Sani, N. A., S. H. P. Hartantyo, and S. J. Forsythe. 2013. Microbiological assessment and evaluation of rehydration instructions on powdered infant formulas, follow-up formulas, and infant foods in Malaysia. J. Dairy Sci. 96:1-8. http://dx.doi.org/10.3168/ jds.2012-5409.

Thatrimontrichai, A., A. Apisarnthanarak, P. Chanvitan, W. Janjindamai, S. Dissaneevate, and G. Maneenil. 2013. Risk factors and outcomes of carbapenem-resistant Acinetobacter baumannii bacteremia in neonatal intensive care unit: A case-case-control study. Pediatr. Infect. Dis. J. 32:140-145.

Wang, M., B. Cao, Q. Gao, Y. Sun, P. Liu, L. Feng, and L. Wang. 2009. Detection of Enterobacter sakazakii and other pathogens associated with infant formula powder by use of a DNA microarray. J. Clin. Microbiol. 47:3178-3184.

Zarrilli, R., A. Di Popolo, M. Bagattini, M. Giannouli, D. Martino, M. Barchitta, A. Quattrocchi, V. D. Iula, C. Luca, A. Scarcella, M. Triassi, and A. Agodi. 2012. Clonal spread and patient risk factors for acquisition of extensively drug-resistant Acinetobacter baumannii in a neonatal intensive care unit in Italy. J. Hosp. Infect. $82: 260-265$. 